\title{
Krein's trace theorem revisited
}

\author{
Denis Potapov, Fedor Sukochev, and Dmitriy Zanin
}

\begin{abstract}
We supply the new proof of Krein's Trace Theorem which does not use complex analysis. Our proof holds for $\sigma$-finite von Neumann algebras $\mathcal{M}$ of type II and unbounded perturbations from the predual of $\mathcal{M}$.
\end{abstract}

Mathematics Subject Classification (2010). 47B10,47A60,47C15.

Keywords. Krein spectral shift function, semifinite von Neumann algebras.

\section{Introduction}

The first attempt to deliver a proof of the existence of the Krein Spectral Shift Function (KrSSF) without using complex analytical facts was made by M. Sh. Birman and M. Z. Solomyak in 1972, [3] (see also [2]). Their method was based on the theory of double operator integrals developed by those authors in [4], [5], and [6]. That attempt led to introducing of an important notion of the spectral averaging measure (see also [18]), but was not successful since the authors of [3] failed to prove the absolute continuity of that measure with respect to Lebesgue measure. The second attempt to deliver such proof is due to D. Voiculescu [22], whose method is based on the usage of the classical Weyl-von Neumann theorem. However, his attempt also failed to recover the full generality of Krein's original result. In our present paper, we combine methods drawn from the double operator integration theory [12], [13], and [17] with Voiculescu's ideas and deliver a rather short and straightforward new proof of Krein's result in full generality. The advantage of our present approach is seen from the following extension.

We deliver the complete proof of the existence of the spectral shift function $\xi_{A, B}$ in the setting when $A$ and $B$ are self-adjoint operators affiliated with a $\sigma$-finite semifinite von Neumann algebra $\mathcal{M}$, whose difference $V:=A-B$ belongs to the predual of $\mathcal{M}$. If $\mathcal{M}$ is a type I factor, then this is precisely Krein's result. For general semifinite von Neumann algebra, however, the earlier attempt based on emulating Krein's complex-analytical proof only yielded the result under the additional restrictive assumption that $V$ is necessarily a trace class perturbation from $\mathcal{M}$ [1]. This extension 
is also inaccessible from the approach chosen in [19], which is also broadly based on [22].

\section{Preliminaries}

Let $H$ be an infinite dimensional Hilbert space and let $\mathscr{L}(H)$ be the algebra of all bounded operators in $H$. In what follows, $\mathcal{M}$ is a von Neumann algebra on $H$, that is a $*$-subalgebra of $\mathscr{L}(H)$ closed in the weak operator topology. The identity in $\mathcal{M}$ is denoted by 1 . We are only interested in semifinite von Neumann algebras, that is those which admit a faithful normal semifinite trace $\tau$. We fix a couple $(\mathcal{M}, \tau)$. A von Neumann algebra is said to be $\sigma$-finite if it admits at most countably many orthogonal projections.

An (unbounded) operator is said to be affiliated with $\mathcal{M}$ if it commutes with every operator in the commutant $\mathcal{M}^{\prime}$ of $\mathcal{M}$. Closed densely defined operator $A$ affiliated with $\mathcal{M}$ is said to be $\tau$-measurable if, for every $\varepsilon>0$, there exists a projection $p \in \mathcal{M}$ such that $\tau(p)<\varepsilon$ and such that $(1-p) H \subset \operatorname{dom}(A)$. The collection of all $\tau$-measurable operators is denoted by $S(\tau)$. The real vector space $S_{h}(\tau)=\left\{A \in S(\tau): A=A^{*}\right\}$ is a partially ordered vector space with the ordering defined by setting $A \geq 0$ if and only if $\langle A \xi, \xi\rangle \geq 0$ for all $\xi \in \mathscr{D}(A)$. The positive cone in $S_{h}(\tau)$ will be denoted by $S(\tau)^{+}$. The positive part $A_{+}$and negative part $A_{-}$ of an operator $A \in S_{h}(\tau)$ are defined by

$$
A_{+}:=\int_{\mathbb{R}} \lambda^{+} d E_{A}(-\infty, \lambda] \text { and } A_{-}:=\int_{\mathbb{R}} \lambda^{-} d E_{A}(-\infty, \lambda]
$$

respectively, where $\lambda^{+}=\max (\lambda, 0)$ and $\lambda^{-}=\max (-\lambda, 0)$ and $E_{A}(-\infty, \lambda]$ is the spectral projection of the self-adjoint operator $A$ corresponding to the interval $(-\infty, \lambda]$. It follows immediately from the spectral theorem that $A=A_{+}-A_{-}$.

The notions of the distribution function $n_{A}, A \in S_{h}(\tau)$ and that of the singular value function $\mu(A), A \in S(\tau)$ are defined as follows

$$
n_{A}(t):=\tau\left(E_{A}(t, \infty)\right), t \in \mathbb{R} \quad \text { and } \quad \mu(t ; A):=\inf \left\{s: n_{|A|}(s) \leq t\right\}, \quad t \geq 0 .
$$

It follows directly that the singular value function $\mu(A)$ is a decreasing, rightcontinuous function on the positive half-line $[0, \infty)$. The trace $\tau$ extends to $S(\tau)^{+}$ as a non-negative extended real-valued functional which is positively homogeneous, additive, unitarily invariant and normal. This extension is given by

$$
\tau(A)=\int_{0}^{\infty} \mu(t ; A) d t, \quad A \in S(\tau)^{+},
$$

and satisfies $\tau\left(A^{*} A\right)=\tau\left(A A^{*}\right)$ for all $A \in S(\tau)$. If $\mathcal{M}=\mathscr{L}(\mathscr{H})$ and $\tau$ is the standard trace, then $S(\tau)=\mathcal{M}$. In this case, an operator $A \in S(\tau)$ is compact if and 
only if $\lim _{t \rightarrow \infty} \mu(t ; A)=0$ and if we set

$$
\mu_{n}(A):=\mu(t ; A), \quad t \in[n, n+1), n=0,1,2, \ldots,
$$

then the sequence $\left\{\mu_{n}(A)\right\}_{n=0}^{\infty}$ is just the sequence of eigenvalues of $|A|$ in nonincreasing order and counted according to multiplicity.

The noncommutative space $\mathscr{L}^{p}=\mathscr{L}^{p}(\mathcal{M}, \tau), 1 \leq p \leq \infty$ is defined as follows

$$
\mathscr{L}^{p}=\left\{A \in S(\tau): \mu(|A|) \in L^{p}:=L^{p}(0, \infty)\right\},
$$

where $L^{p}(0, \infty)$ is the usual Lebesgue space. The space $\mathscr{L}^{p}$ is a linear subspace of $S(\tau)$ and the functional $A \longmapsto\|A\|_{p}:=\left(\tau\left(|A|^{p}\right)\right)^{1 / p}, A \in \mathscr{L}^{p}, 1 \leq p<\infty$ is a norm. For convenience, we set $\left(\mathscr{L}^{\infty},\|\cdot\|_{\infty}\right)=\mathcal{M}$ equipped with the uniform operator norm. We have, in particular, $\|A\|_{p}=\|\mu(A)\|_{p}$ and $\|A B\|_{p},\|B A\|_{p} \leq\|A\|_{p}\|B\|_{\infty}$ for all $A \in \mathscr{L}^{p}, B \in \mathcal{M}, 1 \leq p \leq \infty$ (we denote the norm on $\mathscr{L}^{p}$ and the standard norms on Lebesgue spaces $L^{p}(0, \infty)$ and $L^{p}(\mathbb{R})$ by the same symbol $\|\cdot\|_{p}$ and this should not cause any confusion). We recall the following useful formula

$$
\int_{0}^{\infty} n_{A}(s) d s=\left.s n_{A}(s)\right|_{0} ^{\infty}-\int_{0}^{\infty} s d n_{A}(s)=\tau(A)=\|A\|_{1},
$$

which holds for every $A \in S(\tau)^{+}$. Equipped with the norm $\|\cdot\|_{1}$, the space $\mathscr{L}_{1}$ is a Banach space. It is well known (see e.g. [16]) that $\mathscr{L}_{1}$ is isometric to a predual $\mathcal{M}_{*}$ of the von Neumann algebra $\mathcal{M}$. In what follows, we will need the following result whose proof follows verbatim from that of [21], Lemmata 15 and 16, where this result is established for $s, t>0$.

Lemma 1. Suppose that $\mathcal{M}$ is a finite von Neumann algebra and that $\tau(1)<\infty$. The inequality

$$
n_{A+B}(s+t) \leq n_{A}(s)+n_{B}(t)
$$

holds for all $s, t \in \mathbb{R}$ and all operators $A, B \in S_{h}(\tau)$. If, in addition, we have $A \geq B$, then $n_{A} \geq n_{B}$.

The following Weyl-von Neumann type theorem is at the core of the pure analytical approach of this paper. It is (implicitly) proved in [13]. For the classical Weyl-von Neumann theorem we refer the reader to [9].

Theorem 2. Let $\mathcal{M}$ be a von Neumann algebra equipped with a faithful normal semifinite trace $\tau$. For every $A \in \mathcal{M}$, there exists a sequence of $\tau$-finite projections $p_{n} \uparrow 1$ such that $\left\|\left[A, p_{n}\right]\right\|_{2} \rightarrow 0$ as $n \rightarrow \infty$.

Proof. It follows from Lemma 6.4 in [13] that there exists a net $p_{i}, i \in \mathbb{I}$, of orthogonal projections such that the algebra $p_{i} \mathcal{M} p_{i}$ admits a $\tau$-finite generating projection. Since $\mathcal{M}$ is $\sigma$-finite, it follows that the set $\mathbb{I}$ is, at most, countable. The assertion follows now from Lemma 6.3 in [13]. 
The following two lemmas constitute a small complement to Theorem 2 . These lemmas are at the core of Voiculescu's approach [22]. If $A \in S_{h}(\tau)$, then the projection onto the closure of the range of $|A|$ is called the support of $A$ and is denoted by $\operatorname{supp}(A)$.

Lemma 3. Let $\mathcal{M}$ be a von Neumann algebra equipped with a faithful normal semifinite trace $\tau$. If $p_{n} \uparrow 1$ and if $C \in \mathcal{M}, C^{*}=C$ is such that $\tau(\operatorname{supp}(C))<\infty$, then $\left\|\left[C, p_{n}\right]\right\|_{2} \rightarrow 0$, as $n \rightarrow \infty$.

Proof. Define $A_{n}=\operatorname{supp}(C) p_{n} \operatorname{supp}(C)$. We have $A_{n} \uparrow \operatorname{supp}(C)$ and hence the sequence $A_{n}$ strongly converges to $\operatorname{supp}(C)$. Therefore, $A_{n} C^{2} \rightarrow C^{2}$ and $A_{n} C \rightarrow$ $C$ strongly. By [7], Proposition 2.4.1, we have $\left(A_{n} C\right)^{2} \rightarrow C^{2}$ strongly. Since the trace $\tau$ is strongly continuous (see e.g. [20], Lemma 1.2 and Theorem 1.10) and the algebra $\operatorname{supp}(C) \mathcal{M} \operatorname{supp}(C)$ is finite, it follows that $\tau\left(A_{n} C^{2}\right) \rightarrow \tau\left(C^{2}\right)$ and $\tau\left(\left(A_{n} C\right)^{2}\right) \rightarrow \tau\left(C^{2}\right)$. Due to the equality

$$
\left\|\left[C, p_{n}\right]\right\|_{2}^{2}=-\tau\left(\left[C, p_{n}\right]^{2}\right)=2 \tau\left(A_{n} C^{2}\right)-2 \tau\left(\left(A_{n} C\right)^{2}\right)
$$

we conclude the proof.

Lemma 4. Let $\mathcal{M}$ be a von Neumann algebra equipped with a faithful normal semifinite trace $\tau$. Let $A \in \mathcal{M}$ and let $\left\{p_{n}\right\}_{n \geq 0} \subset \mathcal{M}$ be a sequence of $\tau$-finite projections. If $\left\|\left[A, p_{n}\right]\right\|_{2} \rightarrow 0$ as $n \rightarrow \infty$, then for every $m \geq 1$, we have

$$
\tau\left(\left(p_{n} A p_{n}\right)^{m}-A^{m} p_{n}\right) \rightarrow 0, n \rightarrow \infty
$$

Proof. For $m=1$ the assertion is obvious. For every $m \geq 2$, we have

$$
\begin{aligned}
\left|\tau\left(\left(p_{n} A p_{n}\right)^{m}-A^{m} p_{n}\right)\right|= & \left|\sum_{k=1}^{m-1} \tau\left(p_{n} A^{k}\left(1-p_{n}\right)\left(A p_{n}\right)^{m-k}\right)\right| \leq \\
& \sum_{k=1}^{m-1}\left\|p_{n} A^{k}\left(1-p_{n}\right)\right\|_{2}\left\|\left(1-p_{n}\right) A p_{n}\right\|_{2}\|A\|_{\infty}^{m-k-1} .
\end{aligned}
$$

Observing the equalities

$$
\left(1-p_{n}\right) A p_{n}=\left[A, p_{n}\right] p_{n}, \quad p_{n} A^{k}\left(1-p_{n}\right)=p_{n}\left[p_{n}, A^{k}\right],
$$

and

$$
\left[p_{n}, A^{k}\right]=\sum_{l=0}^{k-1} A^{l}\left[p_{n}, A\right] A^{k-1-l}
$$


we complete the proof as follows

$$
\begin{aligned}
& \left|\tau\left(\left(p_{n} A p_{n}\right)^{m}-A^{m} p_{n}\right)\right| \\
& \quad \leq \sum_{k=1}^{m-1} k\left\|\left[p_{n}, A\right]\right\|_{2}\|A\|_{\infty}^{k-1} \cdot\left\|\left[A, p_{n}\right]\right\|_{2} \cdot\|A\|_{\infty}^{m-k-1} \\
& \quad=\frac{m(m-1)}{2}\left\|\left[A, p_{n}\right]\right\|_{2}^{2}\|A\|_{\infty}^{m-2} .
\end{aligned}
$$

The following result can be found in [17], Corollary 2 and Theorem 4, however it was known much earlier (see e.g. [12], Corollary 7.8, and references therein). Throughout the paper we use the notation $C_{b}(\mathbb{R})$ for the space of all bounded continuous functions on $\mathbb{R}$. The spaces $C_{b}^{1}(\mathbb{R})$ and $C_{b}^{2}(\mathbb{R})$ are defined in a similar manner.

Theorem 5. Let $\mathcal{M}$ be a von Neumann algebra equipped with a faithful normal finite trace $\tau$. If $A, B$ are unbounded self-adjoint operators affiliated with $\mathcal{M}$ such that $A-B \in \mathscr{L}_{1}$, then $f(A)-f(B) \in \mathscr{L}_{1}$ for every $f \in C_{b}^{2}(\mathbb{R})$. Moreover, we have

$$
\|f(A)-f(B)\|_{1} \leq \operatorname{const}\left(\|f\|_{\infty}+\left\|f^{\prime}\right\|_{\infty}+\left\|f^{\prime \prime}\right\|_{\infty}\right)\|A-B\|_{1} .
$$

Observe that, if $A, B$ are unbounded self-adjoint operators affiliated with $\mathcal{M}$ such that $A-B \in \mathscr{L}_{1}$, then

$$
\tau(f(A)-f(B))=\int_{0}^{1} \tau\left(f^{\prime}((1-z) A+z B)(A-B)\right) d z
$$

for every $f \in C_{b}^{2}(\mathbb{R})$. The proof of (3) in the general case is the same as that of [3], equation (2.1).

The class $\boldsymbol{W}_{\mathbf{1}}$. Originally Krein's Trace Theorem (see Theorem 7 below) was proved for the function of class $W_{1}$. That is,

$$
W_{1}=\left\{f \in S^{\prime}(\mathbb{R}): \mathcal{F}\left(f^{\prime}\right) \in L_{1}(\mathbb{R})\right\},
$$

where $S^{\prime}(\mathbb{R})$ is the class of all tempered distributions on $\mathbb{R}, \mathcal{F}$ is the Fourier transform and $L_{1}(\mathbb{R})$ is the Lebesgue space of all integrable functions on $\mathbb{R}$. The class $W_{1}$ is equipped with the semi-norm

$$
\|f\|_{W_{1}}:=\left\|\mathcal{F}\left(f^{\prime}\right)\right\|_{1} .
$$

We need the following simple observation which directly follows from the fact that the class $S(\mathbb{R})$ of all Schwartz functions is dense in $L_{1}(\mathbb{R})$.

Lemma 6. The class of primitives of functions in $S(\mathbb{R})$ is dense in $W_{1}$, that is for every $f \in W_{1}$, there exists a sequence $f_{n} \in S^{\prime}(\mathbb{R})$ such that $f_{n}^{\prime} \in S(\mathbb{R})$ and

$$
\lim _{n \rightarrow \infty}\left\|f-f_{n}\right\|_{W_{1}}=0 .
$$




\section{Krein's theorem in semifinite setting}

The present section proves the following theorem in complete generality. In the setting $\mathcal{M}=\mathscr{L}(\mathscr{H})$ the result is originally due to M.G. Krein [10], Theorem 4.

Theorem 7. Let $\mathcal{M}$ be a $\sigma$-finite von Neumann algebra with a faithful normal semifinite trace $\tau$. If self-adjoint operators $A, B$ affiliated with $\mathcal{M}$ are such that $A-B \in \mathscr{L}_{1}$, then there is a function $\xi=\xi_{A, B} \in L^{1}(\mathbb{R})$ such that

$$
\tau(f(A)-f(B))=\int_{\mathbb{R}} f^{\prime}(s) \xi(s) d s .
$$

for every $f \in W_{1}$.

Proof of Theorem 7. We shall approach the proof of Theorem 7 via step by step relaxing of conditions on the trace $\tau$, function $f$ and the operators $A$ and $B$. This is presented as a series of lemmas from Lemma 8 to Lemma 12. The final extension to the class $W_{1}$ is given in Lemma 13. For reader's convenience, we shall denote the function $\xi_{A, B}$ at different stages with different indices.

We start with rather restrictive case as in the following lemma. The lemma was noted yet by Krein [10] and [11] (see also p. 360 in [1]).

Lemma 8. Suppose that the assumptions of Theorem 7 hold and that $\tau(1)<\infty$. Let $A, B \in \mathcal{M}$ be self-adjoint operators.

(i) Equality (4) holds with $\xi=\xi_{A, B}^{(1)}=n_{A}-n_{B}$. The function $\xi$ is supported on $\left[-\max \left\{\|A\|_{\infty},\|B\|_{\infty}\right\}, \max \left\{\|A\|_{\infty},\|B\|_{\infty}\right\}\right]$.

(ii) Furthermore,

$$
\left\|n_{A}-n_{B}\right\|_{\infty} \leq \tau(\operatorname{supp}(A-B)) \quad \text { and } \quad\left\|n_{A}-n_{B}\right\|_{1} \leq\|A-B\|_{1} .
$$

(iii) If, in addition, $A \geq B$, then $\left\|n_{A}-n_{B}\right\|_{1}=\|A-B\|_{1}$.

Proof. (i) Denote, for brevity, $a=\max \left\{\|A\|_{\infty},\|B\|_{\infty}\right\}$ and fix $\varepsilon>0$. Since $E_{A}(s, \infty)=0$ for $s \geq a+\varepsilon$ and $E_{A}(s, \infty)=1$ for $s \leq-a-\varepsilon$, it follows from the functional calculus that

$$
f(A)=-\int_{-\infty}^{\infty} f(s) d E_{A}(s, \infty)=-\int_{-a-\varepsilon}^{a+\varepsilon} f(s) d E_{A}(s, \infty) .
$$

Taking the trace and integrating by parts, we obtain

$$
\tau(f(A))=-\int_{-a-\varepsilon}^{a+\varepsilon} f(s) d n_{A}(s)=f(-a-\varepsilon)+\int_{-a-\varepsilon}^{a+\varepsilon} f^{\prime}(s) n_{A}(s) d s .
$$


Therefore,

$$
\tau(f(A)-f(B))=\int_{-a-\varepsilon}^{a+\varepsilon} f^{\prime}(s)\left(n_{A}(s)-n_{B}(s)\right) d s .
$$

Since $\varepsilon>0$ is arbitrarily small, the equation (4) follows immediately. Since the function $n_{A}-n_{B}$ is bounded, it follows that it is integrable on the interval $(-a, a)$.

(ii) It is clear that $A \leq B+|A-B|$ and, therefore, by Lemma 1

$$
n_{A}(t) \leq n_{B+|B-A|}(t) \stackrel{(2)}{\leq} n_{B}(t)+n_{|A-B|}(0) .
$$

Similarly, we have $B \leq A+|A-B|$ and, therefore, by Lemma 1

$$
n_{B}(t) \leq n_{A+|B-A|}(t) \stackrel{(2)}{\leq} n_{A}(t)+n_{|A-B|}(0) .
$$

Combining these inequalities, we obtain $\left|n_{A}-n_{B}\right| \leq n_{|A-B|}(0)$, which proves the first inequality. In order to prove the second inequality, observe that

$$
B-A=(B-A)_{+}-(B-A)_{-} .
$$

Set $C:=A+(B-A)_{+}=B+(B-A)_{-}$. We then have $C \geq A$ and $C \geq B$. By Lemma 1 , we have that $n_{C} \geq n_{A}$ and $n_{C} \geq n_{B}$. Obviously,

$$
\left(n_{C}-n_{A}\right)(s)=\left(n_{C+a}-n_{A+a}\right)(s+a), \quad s \in(-\infty, \infty)
$$

and, therefore,

$$
\left\|n_{C}-n_{A}\right\|_{1}=\left\|n_{C+a}-n_{A+a}\right\|_{1}=\int_{0}^{\infty} n_{C+a}(s) d s-\int_{0}^{\infty} n_{A+a}(s) d s .
$$

Since the operators $A+a$ and $C+a$ are positive, it follows now from (1) that

$$
\left\|n_{C}-n_{A}\right\|_{1}=\tau(C+a)-\tau(A+a)=\tau\left((B-A)_{+}\right) .
$$

Similarly, we have

$$
\left\|n_{C}-n_{B}\right\|_{1}=\tau\left((B-A)_{-}\right) .
$$

Hence,

$$
\begin{aligned}
\left\|n_{A}-n_{B}\right\|_{1} & \leq\left\|n_{C}-n_{A}\right\|_{1}+\left\|n_{C}-n_{B}\right\|_{1} \\
& =\tau\left((B-A)_{+}\right)+\tau\left((B-A)_{-}\right)=\|A-B\|_{1} .
\end{aligned}
$$

(iii) If $A \geq B$, then a similar argument shows that

$$
\begin{aligned}
\left\|n_{A}-n_{B}\right\|_{1} & =\left\|n_{A+a}-n_{B+a}\right\|_{1} \\
& =\int_{0}^{\infty} n_{A+a}(s) d s-\int_{0}^{\infty} n_{B+a}(s) d s \\
& =\tau(A+a)-\tau(B+a) \\
& =\tau(A-B) .
\end{aligned}
$$


The key step in our approach is the extension of Lemma 8 to the following lemma via approximation process set out in Theorem 2 and Lemmas 3 and 4.

Lemma 9. Suppose that the assumptions of Theorem 7 hold. If self-adjoint operators $A, B \in \mathcal{M}$ are such that $A \geq B$ and $\tau(\operatorname{supp}(A-B))<\infty$, then (4) holds for every $f(s)=s^{m}, m \in \mathbb{N}$, and for the positive function $\xi=\xi_{A, B}^{(2)}$. We also have

$$
\operatorname{supp}\left(\xi_{A, B}^{(2)}\right) \subset\left[-\max \left\{\|A\|_{\infty},\|B\|_{\infty}\right\}, \max \left\{\|A\|_{\infty},\|B\|_{\infty}\right\}\right]
$$

and $\left\|\xi_{A, B}^{(2)}\right\|_{1}=\|A-B\|_{1}$. If the conditions of Lemma 8 are also met, then $\xi_{A, B}^{(1)}=$ $\xi_{A, B}^{(2)}$.

Proof. By Theorem 2, there exists a family of $\tau$-finite projections $p_{n}, n \geq 0$, such that $p_{n} \uparrow 1$ and such that $\left\|\left[A, p_{n}\right]\right\|_{2} \rightarrow 0$ as $n \rightarrow \infty$. Applying Lemma 3 for $C=B-A$, we infer that $\left\|\left[B-A, P_{n}\right]\right\|_{2} \rightarrow 0$ as $n \rightarrow \infty$. Thus, we also have $\left\|B, p_{n}\right\|_{2} \rightarrow 0$ as $n \rightarrow \infty$. It follows from Lemma 4 that

$$
\tau\left(\left(p_{n} A p_{n}\right)^{m}-A^{m} p_{n}\right) \longrightarrow 0 \text { and } \tau\left(\left(p_{n} B p_{n}\right)^{m}-B^{m} p_{n}\right) \longrightarrow 0,
$$

and so

$$
\begin{aligned}
& \tau\left(\left(p_{n} A p_{n}\right)^{m}-\left(p_{n} B p_{n}\right)^{m}\right)-\tau\left(A^{m}-B^{m}\right) \\
& =\tau\left(\left(p_{n} A p_{n}\right)^{m}-A^{m} p_{n}\right) \\
& \quad-\tau\left(\left(p_{n} B p_{n}\right)^{m}-B^{m} p_{n}\right) \\
& \quad+\tau\left(\left(A^{m}-B^{m}\right)\left(1-p_{n}\right)\right) \longrightarrow 0
\end{aligned}
$$

as $n \rightarrow \infty$, since $p_{n} \uparrow 1$.

Set $a:=\max \left\{\|A\|_{\infty},\|B\|_{\infty}\right\}$. Since $A \geq B$ in $\mathcal{M}$ it follows that $p_{n} A p_{n} \geq$ $p_{n} B p_{n}$ in the algebra $p_{n} \mathcal{M} p_{n}$. In particular, we have $n_{p_{n} A p_{n}} \geq n_{p_{n} B p_{n}}$ for all $n \geq 0$ in the algebra $p_{n} \mathcal{M} p_{n}$. By Lemma 8 (i), for every $n \geq 0$, there exists a positive function $\xi_{n}=\xi_{p_{n} A p_{n}, p_{n} B p_{n}}^{(1)}$ supported on $[-a, a]$ such that

$$
\tau\left(\left(p_{n} A p_{n}\right)^{m}-\left(p_{n} B p_{n}\right)^{m}\right)=\int_{-a}^{a} m s^{m-1} \xi_{n}(s) d s .
$$

By Lemma 8 (ii), we have

$$
\left\|\xi_{n}\right\|_{\infty} \leq \tau(\operatorname{supp}(A-B)) \quad \text { and } \quad\left\|\xi_{n}\right\|_{1} \leq\|A-B\|_{1} .
$$

Since $L_{\infty}[-a, a]$ is a Banach dual for $L_{1}[-a, a]$, it follows from the Banach-Alaoglu theorem that there exists a directed set $\mathbb{I}$ and the mapping $\psi: \mathbb{I} \rightarrow \mathbb{Z}_{+}$such that for every $n \in \mathbb{Z}_{+}$, there exists $i(n) \in \mathbb{I}$ such that $\psi(i)>n$ for $i>i(n)$ and such that the net $\xi_{\psi(i)}, i \in \mathbb{I}$ converges in weak* topology. 
Taking the limit in that topology, we set

$$
\xi_{A, B}^{(2)}=\lim _{i \in \mathbb{I}} \xi_{\psi(i)} .
$$

Therefore,

$$
\begin{aligned}
\int_{-a}^{a} m s^{m-1} \xi_{A, B}^{(2)}(s) d s & =\lim _{i \in I} \int_{-a}^{a} m s^{m-1} \xi_{\psi(i)}(s) d s \\
& =\lim _{i \in I} \tau\left(\left(p_{\psi(i)} A p_{\psi(i)}\right)^{m}-\left(p_{\psi(i)} B p_{\psi(i)}\right)^{m}\right) \\
& =\tau\left(A^{m}-B^{m}\right) .
\end{aligned}
$$

This shows (4) with $f(s)=s^{m}$. The function $\xi_{A, B}^{(2)}$ is positive as a weak ${ }^{*}$-limit of positive functions. In particular, with $m=1$ we have $\left\|\xi_{A, B}^{(2)}\right\|_{1}=\|A-B\|_{1}$. It is clear that this function is supported on $[-a, a]$. This proves all assertions except the last one.

We now suppose that the conditions of Lemma 8 hold and prove that $\xi_{A, B}^{(1)}=\xi_{A, B}^{(2)}$. We have

$$
\begin{aligned}
\int_{\mathbb{R}} m s^{m-1} \xi_{A, B}^{(1)}(s) d s & \stackrel{\text { L. } 8}{=} \tau\left(A^{m}-B^{m}\right) \\
& \stackrel{(5)}{=} \int_{\mathbb{R}} m s^{m-1} \xi_{A, B}^{(2)}(s) d s .
\end{aligned}
$$

Identification now follows from the fact that the polynomials are a separating family of functionals on $L_{1}([-a, a])$.

The following lemma removes the positivity assumption on the operators $A$ and $B$ (although they are still assumed bounded) and the assumption that $\tau(\operatorname{supp}(A-B))$ is finite.

Lemma 10. Suppose that the assumptions of Theorem 7 hold. If $A, B \in \mathcal{M}$ are selfadjoint operators, then (4) holds for every $f \in C_{b}^{2}(\mathbb{R})$ and for the unique compactly supported function $\xi=\xi_{A, B}^{(3)}$. Moreover,

$$
\|\xi\|_{1} \leq\|A-B\|_{1}
$$

and

$$
\int_{\mathbb{R}} \xi(s) d s=\tau(A-B) .
$$

If, in addition, the conditions of Lemma 9 are met, then $\xi_{A, B}^{(2)}=\xi_{A, B}^{(3)}$. 
Proof. Set $C:=A+(B-A)_{+}=B+(B-A)_{-}$. Observe that $C \geq A, C \geq B$ and that $C-A, C-B \in \mathscr{L}_{1}$. Since

$$
\tau(f(A)-f(B))=\tau(f(C)-f(B))-\tau(f(C)-f(A)),
$$

it is sufficient to consider only the case $A \geq B$.

Set $a:=\max \left\{\|A\|_{\infty},\|B\|_{\infty}\right\}$. Let $0 \leq D_{n} \leq A-B$ be such that $D_{n} \uparrow A-B$ and such that $\tau\left(\operatorname{supp}\left(D_{n}\right)\right)<\infty$. The order continuity of $\|\cdot\|_{1}$ (see e.g. [8]) implies that $\left\|B+D_{n}-A\right\|_{1} \rightarrow 0$ as $n \rightarrow \infty$, therefore by Theorem 5 we have

$\left|\tau\left(f\left(B+D_{n}\right)-f(A)\right)\right| \leq \operatorname{const}\left(\|f\|_{\infty}+\left\|f^{\prime}\right\|_{\infty}+\left\|f^{\prime \prime}\right\|_{\infty}\right)\left\|B+D_{n}-A\right\|_{1} \longrightarrow 0$,

as $n \rightarrow \infty$. Since polynomials are dense in $C^{2}[-a, a]$, it follows from (6) and Lemma 9 that

$$
\tau\left(f\left(B+D_{n}\right)-f(B)\right)=\int_{-a}^{a} f^{\prime}(s) \xi_{B+D_{n}, B}^{(2)}(s) d s
$$

for every $f \in C_{b}^{2}(\mathbb{R})$. Hence, we have

$$
\begin{aligned}
& \int_{-a}^{a} f^{\prime}(s) \xi_{B+D_{n}, B+D_{m}}(s) d s \\
& \quad \stackrel{\mathrm{L.} 9}{=} \tau\left(f\left(B+D_{n}\right)-f\left(B+D_{m}\right)\right) \\
& \quad=\tau\left(f\left(B+D_{n}\right)-f(B)\right)-\tau\left(f\left(B+D_{m}\right)-f(B)\right) \\
& \quad \stackrel{(7)}{=} \int_{-a}^{a} f^{\prime}(s)\left(\xi_{B+D_{n}, B}(s)-\xi_{B+D_{m}, B}(s)\right) d s, \quad n \geq m .
\end{aligned}
$$

Since $f^{\prime}$ is an arbitrary $C^{1}$ function on $[-a, a]$, it follows that

$$
\xi_{B+D_{n}, B}^{(2)}-\xi_{B+D_{m}, B}^{(2)}=\xi_{B+D_{n}, B+D_{m}}^{(2)} \geq 0, \quad n \geq m .
$$

Setting $f(s)=s$ in Lemma 9, we obtain that $\left\|\xi_{B+D_{n}, B}^{(2)}\right\|_{1} \leq\left\|D_{n}\right\|_{1}$. Since $\left\|D_{n}\right\|_{1} \leq\|A-B\|_{1}$, it follows from the Monotone Convergence Principle, that the sequence $\xi_{B+D_{n}, B}^{(2)}$ converges in $L^{1}(\mathbb{R})$. Denoting its limit by $\xi_{A, B}^{(3)}$, we obtain

$$
\begin{aligned}
\tau(f(A)-f(B)) & \stackrel{(6)}{=} \lim _{n \rightarrow \infty} \tau\left(f\left(B+D_{n}\right)-f(B)\right) \\
& \stackrel{(7)}{=} \lim _{n \rightarrow \infty} \int_{-a}^{a} f^{\prime}(s) \xi_{B+D_{n}, B}^{(2)}(s) d s \\
& =\int_{-a}^{a} f^{\prime}(s) \xi_{A, B}^{(3)}(s) d s .
\end{aligned}
$$

This proves all the assertions except the last one.

If the conditions of Lemma 9 hold then the identification $\xi_{A, B}^{(2)}=\xi_{A, B}^{(3)}$ can be established similarly to that in Lemma 9. 
The next step in our approach is removing the assumption that the operators $A$ and $B$ are bounded. We remove this assumption in two steps: (i) first we show our construction of a locally integrable $\xi$ for unbounded pair of $A$ and $B$ (see Lemma 11); and (ii) we show that this new $\xi$ is integrable (and positive when $A \geq B$ ); see Lemma 12.

Lemma 11. Suppose that the assumptions of Theorem 7 hold. Then (4) holds with some locally integrable function $\xi_{A, B}^{(4)}$, for every $f \in C_{b}^{2}(\mathbb{R})$ such that the following limits exist

$$
\lim _{s \rightarrow \pm \infty} f(s), \lim _{s \rightarrow \pm \infty} \frac{f^{\prime}(s)}{h^{\prime}(s)}, \lim _{s \rightarrow \pm \infty} \frac{f^{\prime \prime}(s) h^{\prime}(s)-f^{\prime}(s) h^{\prime \prime}(s)}{\left(h^{\prime}(s)\right)^{3}},
$$

where $h$ is a $C^{2}$-bijection $(-\infty, \infty) \rightarrow(a, b)$, for some $a<b \in \mathbb{R}$.

Proof. As in Lemma 10, we may assume that $A \geq B$. Applying Theorem 5 to the operators $A, B$ and to the function $h$, we obtain

$$
\|h(A)-h(B)\|_{1} \leq \text { const } \cdot\|A-B\|_{1} .
$$

We shall prove the assertion for the case $a=-1, b=1$ only. We now define $g \in$ $C^{2}(-1,1)$ by setting $g:=f \circ h^{-1}$. Condition (8) ensures that $g \in C^{2}[-1,1]$ and, therefore, $g$ extends to a function $g \in C_{b}^{2}(\mathbb{R})$. Since $h$ is a $C^{2}$-bijection $(-\infty, \infty) \rightarrow$ $(a, b)$, the operators $h(A)$ and $h(B)$ are bounded, therefore, applying Lemma 10 to the operators $h(A)$ and $h(B)$, we now obtain

$$
\begin{aligned}
\tau(f(A)-f(B)) & =\tau(g(h(A))-g(h(B))) \\
& =\int_{\mathbb{R}} g^{\prime}(s) \xi_{h(A), h(B)}^{(3)}(s) d s \\
& =\int_{\mathbb{R}} f^{\prime}(u) \xi_{h(A), h(B)}^{(3)}(h(u)) d u,
\end{aligned}
$$

where in the last step we substituted $s=h(u)$. This proves (4). Since the left hand side does not depend on $h$ and since $f^{\prime}$ is sufficiently arbitrary, it follows that the expression $\xi_{h(A), h(B)}^{(3)} \circ h$ does not depend on $h$. So we define

$$
\xi_{A, B}^{(4)}:=\xi_{h(A), h(B)}^{(3)} \circ h .
$$

The local integrability of $\xi_{A, B}^{(4)}$ follows from the integrability of $\xi_{h(A), h(B)}^{(3)}$ combined with the assumption that $h$ is a $C^{2}$-bijection.

If $A$ and $B$ are bounded, then by using the trace formula for the left hand side of (9), we obtain

$$
\int_{\mathbb{R}} f^{\prime}(u) \xi_{A, B}^{(3)} d u=\tau(f(A)-f(B))=\int_{\mathbb{R}} f^{\prime}(u) \xi_{A, B}^{(4)}(u) d u .
$$

The latter implies that $\xi_{A, B}^{(3)}=\xi_{A, B}^{(4)}$ in this case. 
Lemma 12. Suppose that the assumptions of Theorem 7 hold. Equality (4) holds with $\xi_{A, B}^{(4)}$ of Lemma 11, for every $f \in S^{\prime}(\mathbb{R})$ such that $f^{\prime} \in S(\mathbb{R})$. Moreover, $\xi_{A, B}^{(4)} \in L_{1}(\mathbb{R})$. If $A \geq B$, then we also have $\xi_{A, B}^{(4)} \geq 0$.

Proof. As in the preceding lemmas, it is sufficient to consider only the case when $A \geq B$. In this case, we shall show that the function $\xi_{A, B}^{(4)}$ of Lemma 11 is positive and integrable.

We shall show positivity first. Let $\alpha>0$ and let $a<b \in \mathbb{R}$. Define the function $h_{a, b, \alpha}$ by setting

$$
h_{a, b, \alpha}(t):= \begin{cases}t, & t \in[a, b], \\ \frac{\alpha(t-b)}{\left(\alpha^{2}+(t-b)^{2}\right)^{1 / 2}}+b, & t>b, \\ \frac{\alpha(t-a)}{\left(\alpha^{2}+(t-a)^{2}\right)^{1 / 2}}+a, & t<a .\end{cases}
$$

Observe that the function $h_{a, b, \alpha}$ is continuous and

$$
\lim _{t \rightarrow+\infty} h_{a, b, \alpha}(t)=\alpha+b \quad \text { and } \quad \lim _{t \rightarrow-\infty} h_{a, b, \alpha}(t)=a-\alpha
$$

Computing the derivative

$$
h_{a, b, \alpha}^{\prime}(t)= \begin{cases}1, & t \in[a, b], \\ \frac{\alpha^{3}}{\left(\alpha^{2}+(t-b)^{2}\right)^{3 / 2}}, & t>b, \\ \frac{\alpha^{3}}{\left(\alpha^{2}+(t-a)^{2}\right)^{3 / 2}}, & t<a,\end{cases}
$$

we have that the function $h_{a, b, \alpha}$ is a strictly increasing function on $\mathbb{R}$ and therefore it is a $C^{2}$-bijection $(-\infty, \infty) \rightarrow(a-\alpha, b+\alpha)$. Since $f^{\prime}$ is a Schwartz function, it is not difficult to verify that $h_{a, b, \alpha}$ satisfies the condition (8) in Lemma 11.

If $\xi_{A, B}^{(4)}$ is from Lemma 11 , then

$$
\xi_{A, B}^{(4)}=\xi_{h_{a, b, \alpha}^{(3)}(A), h_{a, b, \alpha}(B)}^{\circ} h_{a, b, \alpha}
$$

Combining Theorem 5 with the well known fact that $\tau(C D) \geq 0$ when $0 \leq C \in$ $\mathcal{M}$ and $0 \leq D \in \mathscr{L}^{1}$, we obtain the following estimate

$$
\tau\left(h_{a, b, \alpha}(A)-h_{a, b, \alpha}(B)\right)=\int_{0}^{1} \tau\left(h_{a, b, \alpha}^{\prime}((1-z) A+z B)(A-B)\right) d z \geq 0 .
$$


Furthermore, by Lemma 10 (applied to $h_{a, b, \alpha}(A), h_{a, b, \alpha}(B)$ and $f(s)=s$ ) and substituting $s=h_{a, b, \alpha}(u)$, we obtain

$$
\begin{aligned}
\tau\left(h_{a, b, \alpha}(A)-h_{a, b, \alpha}(B)\right) & =\int_{a-\alpha}^{b+\alpha} \xi_{h_{a, b, \alpha}^{(3)}(A), h_{a, b, \alpha}(B)}(s) d s \\
& =\int_{\mathbb{R}} \xi_{h_{a, b, \alpha}^{(3)}(A), h_{a, b, \alpha}(B)}\left(h_{a, b, \alpha}(u)\right) h_{a, b, \alpha}^{\prime}(u) d u \\
& =\int_{\mathbb{R}} \xi_{A, B}^{(4)}(u) h_{a, b, \alpha}^{\prime}(u) d u .
\end{aligned}
$$

In particular, the function $\xi_{A, B}^{(4)} h_{a, b, \alpha}^{\prime}$ is integrable. Since $h_{a, b, \alpha}^{\prime} \rightarrow \chi_{(a, b)}$ almost everywhere as $\alpha \rightarrow 0$ and since $h_{a, b, \alpha}^{\prime} \leq h_{a, b, 1}^{\prime} \in L^{1}(\mathbb{R})$ when $\alpha<1$, it follows from the Dominated Convergence Principle that

$$
\int_{a}^{b} \xi_{A, B}^{(4)}(u) d u=\lim _{\alpha \rightarrow 0} \int_{\mathbb{R}} \xi_{A, B}^{(4)}(u) h_{a, b, \alpha}^{\prime}(u) d u \geq 0 .
$$

Since the latter inequality holds for arbitrary $a, b$, it follows that $\xi_{A, B}^{(4)} \geq 0$.

Now we show the integrability of $\xi_{A, B}^{(4)}$ on $\mathbb{R}$. Consider the function

$$
h_{\alpha}: \mathbb{R} \longrightarrow(-\alpha, \alpha)
$$

given by

$$
h_{\alpha}(s)=\frac{\alpha s}{\left(\alpha^{2}+s^{2}\right)^{\frac{1}{2}}}, \quad s \in \mathbb{R} .
$$

Repeating the same arguments that used above for the function $h_{a, b, \alpha}$, we obtain that the function $h_{\alpha}$ is the $C^{2}$-bijection $\mathbb{R} \rightarrow(-1,1)$ and satisfies the condition (8) in Lemma 11. If $\xi_{A, B}^{(4)}$ is from Lemma 11 , then

$$
\xi_{A, B}^{(4)}=\xi_{h_{\alpha}(A), h_{\alpha}(B)}^{(3)} \circ h_{\alpha} .
$$

By Lemma 10 (applied to the operators $h_{\alpha}(A)$ and $h_{\alpha}(B)$ ) we have

$$
\begin{aligned}
\tau\left(h_{\alpha}(A)-h_{\alpha}(B)\right) & =\int_{-\alpha}^{\alpha} \xi_{h_{\alpha}(A), h_{\alpha}(B)}^{(3)}(s) d s \\
& =\int_{\mathbb{R}} \xi_{h_{\alpha}(A), h_{\alpha}(B)}^{(3)}\left(h_{\alpha}(t)\right) h_{\alpha}^{\prime}(t) d t \\
& =\int_{\mathbb{R}} \xi_{A, B}^{(4)}(t) h_{\alpha}^{\prime}(t) d t .
\end{aligned}
$$


We have

$$
\begin{aligned}
\left|\int_{-\infty}^{\infty} \xi_{A, B}^{(4)}(t) \cdot h_{\alpha}^{\prime}(t) d t\right| & =\left|\tau\left(h_{\alpha}(A)-h_{\alpha}(B)\right)\right| \\
& \leq\left\|h_{\alpha}(A)-h_{\alpha}(B)\right\|_{1} \\
& \leq \text { const }\|A-B\|_{1},
\end{aligned}
$$

where the last estimate follows from Theorem 5, applied to operators $\alpha^{-1} A$ and $\alpha^{-1} B$ and function $h_{1}$. Observe that the constant in the latter estimate is independent of $\alpha$. Since $h_{\alpha}^{\prime} \uparrow 1$ when $\alpha \rightarrow \infty$, we infer from the Monotone Convergence Principle (which is applicable since $\xi_{A, B}^{(4)} \geq 0$ ) that $\xi_{A, B}^{(4)}$ is integrable.

Finally we give simple extension of Lemma 12 to the class $W_{1}$. The extension is based on our earlier observation in Lemma 6.

Lemma 13. Suppose that the assumptions of Theorem 7 hold. The trace formula (4) holds for every $f \in W_{1}$.

Proof. Fix $f \in W_{1}$. By Lemma 6, there is a sequence $f_{n} \in S^{\prime}(\mathbb{R})$ such that $f_{n}^{\prime} \in$ $S(\mathbb{R})$ and such that

$$
\lim _{n \rightarrow \infty}\left\|f-f_{n}\right\|_{W_{1}}=0 .
$$

Applying Lemma 12 with $\xi=\xi_{A, B}^{(4)}$ to every $f_{n}$, we have

$$
\tau\left(f_{n}(A)-f_{n}(B)\right)=\int_{\mathbb{R}} f_{n}^{\prime}(s) \xi(s) d s .
$$

Since

$$
\left\|f^{\prime}-f_{n}^{\prime}\right\|_{\infty} \leq\left\|\mathcal{F}\left(f^{\prime}-f_{n}^{\prime}\right)\right\|_{1}=\left\|f-f_{n}\right\|_{W_{1}} \rightarrow 0,
$$

we have the convergence of the right hand side of (10)

$$
\lim _{n \rightarrow \infty} \int_{\mathbb{R}} f_{n}^{\prime}(s) \xi(s) d s=\int_{\mathbb{R}} f^{\prime}(s) \xi(s) d s .
$$

On the other hand, the convergence $\left\|f^{\prime}-f_{n}^{\prime}\right\|_{\infty} \rightarrow 0$ guarantees

$$
\lim _{n \rightarrow \infty}\left\|f_{n}^{\prime}\left(C_{t}\right)-f^{\prime}\left(C_{t}\right)\right\|_{\infty}=0,
$$

where $C_{t}=t A+(1-t) B$, uniformly over $t \in(0,1)$. Thus, we arrive at the convergence of the left hand side of (10) as follows

$$
\begin{aligned}
\lim _{n \rightarrow \infty} \tau\left(f_{n}(A)-f_{n}(B)\right) & =\lim _{n \rightarrow \infty} \int_{0}^{1} \tau\left(f_{n}^{\prime}\left(C_{t}\right)(A-B)\right) d t \\
& =\int_{0}^{1} \tau\left(f^{\prime}\left(C_{t}\right)(A-B)\right) d t \\
& =\tau(f(A)-f(B)),
\end{aligned}
$$


where

$$
C_{t}=t A+(1-t) B
$$

Remark 14. We observe that Theorem 7 and the trace formula (4) can be further extended from the class of functions $W_{1}$ to the homogeneous Besov class $\tilde{B}_{\infty 1}^{1}$ (see e.g. [15]). In the case when $\mathcal{M}=\mathscr{L}(H)$, such an extension is performed in [15], in the general case the argument is exactly the same as in [15]. We omit further details.

\section{References}

[1] N. Azamov, P. Dodds, and Sukochev, The Krein spectral shift function in semifinite von Neumann algebras. Integral Equations Operator Theory 55 (2006), 347-362. MR 2244193 Zbl 1105.46043

[2] M. S. Birman and A. B. Pushnitski, Spectral shift function, amazing and multifaceted. Integral Equations Operator Theory 30 (1998), 191-199. Dedicated to the memory of Mark Grigorievich Krein (1907-1989). MR 1607900 Zbl 0907.47014

[3] M. Š. Birman and M. Z. Solomjak, Remarks on the spectral shift function. Zap. Naučn. Sem. Leningrad. Otdel. Mat. Inst. Steklov. (LOMI) 27 (1972), 33-46. Boundary value problems of mathematical physics and related questions in the theory of functions 6 . In Russian. English transl., J. Sov. Math. 3 (1975), 408-419. MR 0315482 Zbl 0329.47009 Zbl 0336.47017 (transl.)

[4] M. Š. Birman and M. Z. Solomjak, Double Stieltjes operator integrals. Problemy Mat. Fiz. 1 (1966), 33-67. In Russian. MR 0209872 Zbl 0161.34602

[5] M. Š. Birman and M. Z. Solomjak, Double Stieltjes operator integrals II. Problemy Mat. Fiz. 2 (1967), 26-60. In Russian. MR 0182.46202 Zbl 0234304

[6] M. Š. Birman and M. Z. Solomjak, Double Stieltjes operator integrals III. Problemy Mat. Fiz. 6 (1973), 27-53. IN Russian. MR 0348494 Zbl 0281.47013

[7] O. Bratteli and D. Robinson, Operator algebras and quantum statistical mechanics. 1. $C^{*}$ - and $W^{*}$-algebras, symmetry groups, decomposition of states. Second ed. Texts and Monographs in Physics. Springer Verlag, New York, N.Y., 1987. MR 0887100 Zbl 0905.46046

[8] V. I. Chilin and F. A. Sukochev, Weak convergence in non-commutative symmetric spaces. J. Oper. Theory 31 (1994), 35-65. MR 1316983 Zbl 0836.46057

[9] K. Davidson, $C^{*}$-algebras by example. Fields Institute Monographs 6. American Mathematical Society, Providence, R.I., 1996. MR 1402012 Zbl 0956.46034

[10] M. Krein, On the trace formula in perturbation theory. Mat. Sbornik N.S. 33(75) (1953), 597-626. MR 0060742

[11] M. Krein, On some new investigations in perturbation theory. In First mathematical summer school (Kanev, June-July 1963). Part I. Akademija Nauk Ukrainskoi SSR. Institut Matematiki. Naukova Dumka, Kiev, 1964, 104-183. In Russian. MR 0184819 (collection) Zbl 1245.00035 (collection) 
[12] B. de Pagter and F. Sukochev, Differentiation of operator functions in non-commutative $L_{p}$-spaces. J. Funct. Anal. 212 (2004), 28-75. MR 2065237 Zbl 1075.46054

[13] B. de Pagter, F. Sukochev, and H. Witvliet, Double operator integrals. J. Funct. Anal. 192 (2002), 52-111. MR 1918492 Zbl 1079.47502

[14] V. V. Peller, An extension of the Koplienko-Neidhardt trace formulae. J. Funct. Anal. 221 (2005), 456-481. MR 2124872 Zbl 1067.47019

[15] V. V. Peller, Hankel operators in the perturbation theory of unbounded selfadjoint operators. In C. Sadosky (ed.), Analysis and partial differential equations. A collection of papers dedicated to M. Cotlar. Lecture Notes in Pure and Applied Mathematics 122. Marcel Dekker, New York, N.Y., 1990, 529-544. MR 1044807 MR 1044775 (collection) Zbl 0716.47015 Zbl 0684.00014 (collection)

[16] G. Pisier and Q. Xu, Non-commutative $L_{p}$-spaces. In W. B. Johnson and J. Lindenstrauss (eds.), Handbook of the geometry of Banach spaces. Vol. 2. North-Holland, Amsterdam, 2003, 1459-1517. MR 1999201 MR 1999613 (collection) Zbl 1046.46048 Zbl 1013.46001 (collection)

[17] D. Potapov and F. Sukochev, Unbounded Fredholm modules and double operator integrals. J. Reine Angew. Math. 626 (2009), 159-185. MR 2492993 Zbl 1189.46063

[18] B. Simon, Spectral averaging and the Krein spectral shift. Proc. Amer. Math. Soc. 126 (1998), 1409-1413. MR 1443857 Zbl 0892.47021

[19] K. Sinha and A. Mohapatra, Spectral shift function and trace formula. Proc. Indian Acad. Sci. 104 (1994), 819-853. MR 1319886 Zbl 0821.47005

[20] Ş. Strătilă and L. Zsidó, Lectures on von Neumann algebras. Editura Academiei, Bucharest, 1979. MR 0526399 Zbl 0391.46048

[21] F. Sukochev and D. Zanin, Johnson-Schechtman inequalities in the free probability theory. J. Funct. Anal. 263 (2012), 2921-2948. MR 2973330 Zbl 1264.46046

[22] D. Voiculescu, On a trace formula of M. G. Krein. In H. Helson, B. Szőkefalvi-Nagy F.-H. Vasilescu, D. Voiculescu and Gr. Arsene (eds.), Operators in indefinite metric spaces, scattering theory and other topics. Papers from the tenth international conference on operator theory held in Bucharest, August 26-September 5, 1985. Operator Theory: Advances and Applications 24. Birkhäuser Verlag, Basel etc., 1987, 329-332. MR 0903085 MR 0903059 (collection) Zbl 0647.47019 Zbl 0614.00015 (collection)

Received June 26, 2013; revised July 25, 2013

Denis Potapov, School of Mathematics and Statistics, University of New South Wales, Kensington, NSW, Australia

E-mail: d.potapov@unsw.edu.au

Fedor Sukochev, School of Mathematics and Statistics, University of New South Wales, Kensington, NSW, Australia

E-mail: f.sukochev@unsw.edu.au

Dmitriy Zanin, School of Mathematics and Statistics, University of New South Wales, Kensington, NSW, Australia

E-mail: d.zanin@unsw.edu.au 